CI) - 0.6 (0.3-1.20)] (GRADE - Very low). Levetiracetam resulted in a lower risk of short-term adverse events compared to phenobarbital [RR (95\% CI) - $0.24(0.06-0.92)]$ (GRADE - Moderate).

Conclusions Very low-quality evidence suggests that levetiracetam might not be more effective than phenobarbital. Moderate quality evidence indicates levetiracetam is associated with a lower risk of adverse events.

\section{AN AUDIT OF THE TIME FROM BIRTH TO FIRST MILK FEED IN PRETERM INFANTS LESS THAN 32 WEEKS GESTATION AND OF LOW-BIRTH-WEIGHT INFANTS UNDER 1500G}

Natasha Ramsden. UK

10.1136/bmjpo-2021-RCPCH.104

Background There are many benefits to starting breast milk feeds early for both preterm and low-birth-weight infants including: the immunological benefits of receiving breast milk, potential to establish full enteral feeds earlier and lowering the risk of necrotising enterocolitis.

Objectives Trust guidelines state that all infants should receive colostrum in the first day of life, preferably within the first 6 hours. The objectives of this audit were to evaluate whether this is achieved in infants $<32$ weeks gestation or with birth weights $<1500 \mathrm{~g}$ and to look at the impact of gestation on the time from birth to first milk feed.

Methods Inclusion criteria were infants born $<32$ weeks gestation or with a birthweight of $<1500 \mathrm{~g}$. The length of time from birth to first milk feed in hours (including either colostrum mouth care or first enteral feed) was evaluated on a total of 52 inpatients on the neonatal unit. Data was initially collected in December 2018 on 24 infants. This was followed by interventions including: education of parents and the multidisciplinary team about the importance of the early colostrum administration and collaboration between the neonatal and maternity teams to provide expressing packs to mother's predelivery. Further data was then collected on 28 infants in July 2020, all born during the first wave of the COVID-19 pandemic. The data from 2018 and 2020 were compared and the impact of gestation was analysed by comparing infants born $<27$ weeks and $\geq 27$ weeks.

Results In $100 \%$ of infants in the study the first milk received was human milk (either mother's own milk or donor breast milk) and all infants received their first milk within 96 hours of birth. Overall, 19.2\% of infants received their first milk within 6 hours of birth and $63.5 \%$ within 24 hours. When comparing the 2018 and 2020 data, a similar percentage of infants received their first milk feed in $<24$ hours: $62.5 \%$ in 2018 and $64.3 \%$ in 2020 , and at $<6$ hours: $20.8 \%$ in 2018 and $17.9 \%$ in 2020 . Infants $\geq 27$ weeks received their first milk feed sooner than infants $<27$ weeks. In extreme preterm infants $<27$ weeks, $10 \%$ received their first milk feed in $<6$ hours and $55 \%$ in $<24$ hours. Comparably in preterm infants $\geq 27$ weeks, $25 \%$ received their first milk feed in $<6$ hours and $68.8 \%$ in $<24$ hours. In both groups, $90 \%$ of infants received their first milk feed within 48 hours of birth.

Conclusions The results in 2018 and 2020 were similar, suggesting that the interventions made during this audit cycle had no observed increase in delivery efficiency of the first milk feed. The second cycle of the audit was carried out during the COVID-19 pandemic but even with many restrictions there has been no observed adverse impact on the delivery of the first milk to this group of neonates. Future focus should improve the prompt delivery of the first milk feed for extreme preterm neonates through discussing expressing colostrum with mother's pre-delivery and giving colostrum mouthcare early to these infants.

\section{LUDWIG'S ANGINA-A CASE REPORT}

Anupama Mallappa, Derek Veitch. UK

\subsection{6/bmjpo-2021-RCPCH.105}

Background Ludwig's angina was first reported by Wilhelm Frederick von Ludwig in 1836. It is a severe and quickly spreading cellulitis characterized by significant involvement of the sub-mandibular, sublingual and occasionally sub-mental space. This condition is potentially fatal if not treated aggressively and promptly due to the cellulitis leading to airway compromise. Prior to antibiotics in the middle of the 20th century, mortality rates were more than $50 \%$.

Most cases of Ludwig's angina documented occur more commonly in adult males and secondary to dental infection (70-90\%) with mandibular molars (unilateral or bilateral involvement) as the main cause. When Ludwig's angina occurs in the paediatric population, odontogenic cause is only $50 \%$. Some reported causes include salivary gland infection due to sialadenitis, oral tumour infection and infection following intraoral soft tissues surgery. A precipitating aetiology may be difficult to determine, the most frequently involved microorganism is streptococci and staphylococci species. Diagnosis of the condition is normally made through history and physical examination, other findings include fever and raised white cell count and raised CRP.

Objectives The patient presented here shows a severe case of a Ludwig's angina found in a healthy adolescent male, an age group in which the condition is less common.

Methods electronic case records were used to collect data

Results 13-year-old boy presented with increased facial swelling, drooling, swollen protruding tongue, unable to speak, soft stridor and unable to open mouth.

Had had root canal treatment 7 days prior and had also attended A\&E 2 days previously with fever, pain on swallowing associated with puffiness around cheeks and eyes. He was assessed and sent home on oral antibiotics.

He had no significant past medical history (extraction of 10 deciduous teeth at $7 \mathrm{yrs}$ ).

On presentation to A\&E he had a obstructed airway, maintaining saturations 96\% room air, febrile, he was tachyopneic, tachycardiac(122/min) and hypertensive (113/99 $\mathrm{mmHg}) . \mathrm{He}$ was managed with nebulized adrenaline, dexamethasone and IV antibiotics. Clinical diagnosis was Ludwig's angina

He underwent awake fibro-optic intubation, in theatre he underwent dental extraction LL6 (noted to have copious pus) +UL6, underwent tracheostomy for airway management.

Was transferred to HDU post procedure. CT scan showed fluid collection containing flecks of air within the left parapharyngeal space, extending antero-superiorly to left postnasal space, laterally insinuating between the masseter and left medial pterygoid muscle. Posteriorly, extending to lie just below left bony external acoustic canal. 
Had a stormy post- operative course with two incidents of peri-arrest due to blocked tracheostomy. These two incidents caused significant anxiety and distress for the child, for which he received psychology input. Decannulation done day 5 postop, discharged home on post-op day 10, with a course oral antibiotics.

Conclusions The present case describes a typical but severe case of Ludwig's angina in a relatively atypical age group. This is a life -threatening condition, which requires early intervention along with a multidisciplinary team input. While relatively uncommon in the paediatric population, clinicians should be aware and understand the urgency of immediate treatment when it does present.

\section{BONE PROFILE IN LOW BIRTH WEIGHT BABIES: WHAT WE LEARN}

Hnin Pwint Oo, Nyan Sin Htun, Nay Aung, Nilar Lwin. Myanmar

\subsection{6/bmjpo-2021-RCPCH.106}

Background Bone profile in low birth weight (LBW) babies is important for monitoring of metabolic bone disease(MBD). $\mathrm{MBD}$ is a clinically heterogeneous group of disease with commonly reversible clinical presentation and manifestation when the underlying defect has been treated.

Objectives This study was aimed to find out biochemical profile in LBW babies and association between bone profile and gestational age of LBW.

Methods A cross-sectional descriptive study was done in all LBW infants at 8 weeks of age within one year period. Infants with less than 28 weeks of gestation, infants with insufficient perinatal records and those with suspicion of renal, hepatic, gastrointestinal and thyroid diseases were excluded.

Results Total 60 LBW (45 preterm and 15 small for gestational age, SGA) babies were included. Biochemical parameters of bone profile were shown in table 1 . Sixteen babies $(26.7 \%)$ were found MBD, comprising $68.7 \%$ of preterm and $31.3 \%$ of SGA infants. The mean serum calcium level was not statistically significant (table2) whereas mean serum phosphate and alkaline phosphatase levels were significantly different between low birth weight infants with MBD and those without MBD (table $3 \& 4$ ).

Abstract 196 Table 1 Biochemical parameters of LBW

\begin{tabular}{lllll}
\hline Variables & Mean & Standard deviation & Minimum & Maximum \\
\hline Calcium(mg/dl) & 7.12 & 0.524 & 8.28 & 10.96 \\
Phosphate(mg/dl) & 2.41 & 0.722 & 1.85 & 11.64 \\
Alkaline phosphatase(IU/L) & 400 & 16 & 100 & 700 \\
\hline
\end{tabular}

Abstract 196 Table 2 Association between serum calcium and gestational age of LBW

\begin{tabular}{|c|c|c|c|c|c|c|c|}
\hline \multirow[t]{2}{*}{ Calcium } & \multicolumn{4}{|c|}{ Gestational age(week) } & \multirow{2}{*}{$\begin{array}{l}\text { Total } \\
-(\%)\end{array}$} & \multirow{2}{*}{$\begin{array}{l}\text { Chi- } \\
\text { square }\end{array}$} & \multirow{2}{*}{$\begin{array}{l}\mathrm{p} \text { - } \\
\text { value }\end{array}$} \\
\hline & $28-31(\%)$ & $31-34(\%)$ & $34-37(\%)$ & $37-40(\%)$ & & & \\
\hline Low & 25 & 31.25 & 31.25 & 12.5 & 100 & 3.63 & 0.727 \\
\hline Normal & 12.2 & 26.82 & 36.58 & 24.4 & 100 & & \\
\hline High & 33.3 & 0 & 33.3 & 33.4 & 100 & & \\
\hline
\end{tabular}

Abstract 196 Table 3 Association between serum phosphate and gestational age of LBW

\begin{tabular}{|c|c|c|c|c|c|c|c|}
\hline \multirow[t]{2}{*}{ Phosphate } & \multicolumn{4}{|c|}{ Gestational age(week) } & \multirow{2}{*}{$\begin{array}{l}\text { Total } \\
-(\%)\end{array}$} & \multirow{2}{*}{$\begin{array}{l}\text { Chi- } \\
\text { square }\end{array}$} & \multirow{2}{*}{$\begin{array}{l}\mathrm{p} \text { - } \\
\text { value }\end{array}$} \\
\hline & $28-31(\%)$ & $31-34(\%)$ & $34-37(\%)$ & $37-40(\%)$ & & & \\
\hline Low & 50 & 12.5 & 12.5 & 25 & 100 & 35.828 & 0.000 \\
\hline Normal & 27.77 & 0 & 72.23 & 0 & 100 & & \\
\hline High & 2.9 & 44.11 & 20.59 & 32.4 & 100 & & \\
\hline
\end{tabular}

Abstract 196 Table 4 Association between serum alkaline phosphatase and gestational age of LBW

\begin{tabular}{llllllll}
\hline Alkaline & \multicolumn{2}{l}{ Gestational age(week) } & & Total & Chi- & p- \\
phosphatase & \multicolumn{2}{l}{ square } & value \\
\cline { 2 - 7 } & $28-31(\%)$ & $31-34(\%)$ & $34-37(\%)$ & $37-40(\%)$ & & & \\
\hline Low & 0 & 0 & 0 & 0 & 0 & 13.918 & 0.003 \\
Normal & 0 & 0 & 88.89 & 11.11 & 100 & & \\
High & 19.6 & 31.37 & 25.5 & 23.53 & 100 & & \\
\hline
\end{tabular}

Conclusions MBD is more common in preterm babies than SGA babies. Serum calcium does not correlate well with gestational age of low birth weight infants where as serum phosphate and alkaline phosphatase have significant correlation. Diagnosis of $\mathrm{MBD}$ can be suspected on the abnormality of biochemical data, especially serum phosphate and alkaline phosphatase levels. Thus, measurement of biochemical bone profile has been recommended for early detection of MBD in high risk infants.

\section{SPECTRUM OF SYMPTOMS IN COVID-19 PAEDIATRIC PATIENTS ACROSS FOUR DISTRICT GENERAL HOSPITALS IN YORKSHIRE}

Sharon Chan, Rajeeva Singh, Alexander-John Shuttleworth. UK

\subsection{6/bmjpo-2021-RCPCH.107}

Background Despite increasing reports and studies about COVID-19 in children over the last nine months, there is still much to be discovered about the true incidence, clinical presentations and transmission rates in children. Fever and respiratory symptoms remain the main clinical presentations in COVID-19 positive children. Symptoms of COVID-19 in the paediatric population appear to be less severe compared to those experienced in the adult population and there is a low case fatality rate for COVID-19 in children. More recently there have been increasing reports of children with Paediatric multisystem inflammatory syndrome temporally associated with COVID-19 (PIMS).

Objectives The objectives of this study were to evaluate the spectrum of clinical presentations and evolution of clinical symptoms, management and outcomes of children with laboratory confirmed COVID-19 who presented to secondary care to help guide future management of these patients. We also describe two cases of Paediatric multisystem inflammatory syndrome temporally associated with COVID-19 (PIMS).

Methods Our study is a retrospective observational study which identified 21 patients aged 1 month to 18 years with positive COVID-19 PCR and 2 patients with PIMS over a 10 